\title{
Medição de blindagem eletromagnética adaptada à realidade dos Laboratórios de Eletromagnetismo
}

\author{
Sandro Magalhães Malta, Julião César Moreira Sales, \\ Maria Inês Martins, Ana de Oliveira Rodrigues
}

\begin{abstract}
Resumo-Este trabalho apresenta um procedimento para avaliação de blindagem eletromagnética através de medição de campos eletromagnéticos em laboratório, baseado nas normas especificadas pelo padrão IEEE Std299-1997, revisado em 2006, "IEEE Standard Method for Measuring the Effectiveness of Electromagnetic Shielding Enclosures" [IEEE, 1997] [IEEE, 2007]. Adaptações para a realidade dos laboratórios de Eletromagnetismo, em particular do Centro Universitário de Belo Horizonte (UNI-BH) e da Pontifícia Universidade Católica de Minas Gerais (PUC Minas) são propostas, como alternativas de baixo custo aos materiais e métodos sugeridos pela norma. São apresentadas as validações das medições através de comparação com solução analítica. O comportamento da blindagem medida com este procedimento mostrase coerente, evidenciando a dificuldade de blindagem de campos de baixa frequiência e o aumento da eficiência da blindagem com o aumento da freqüência. Conclui-se que o procedimento desenvolvido mostra-se como uma alternativa de baixo custo aos métodos apresentados nos padrões internacionais.
\end{abstract}

palavras-chave - blindagem, campos eletromagnéticos, medições laboratoriais.

Abstract-This work presents a procedure to evaluate electromagnetic shielding efficiency using field measurements of electromagnetic fields based on IEEE Std-299. 1997 and its revised version of 2006, "IEEE Standard Method for Measuring the Effectiveness of Electromagnetic Shielding Enclosures" [IEEE, 1997] [IEEE, 2007]. Adaptations to resources commonly available at Electromagnetic Laboratories, especially the laboratories at Centro Universitário de Belo Horizonte (UNI-BH) and at Pontifícia Universidade Católica de Minas Gerais (PUC

Sandro Magalhães Malta é Engenheiro de Telecomunicações, formado pela PUC Minas, atualmente fazendo mestrado em Engenharia Elétrica na PUC Minas. sandrommalta@yahoo.com.br.

Julião César Moreira Sales é Engenheiro de Telecomunicações, formado pelo UNI-BH. juliaosales@ hotmail.com.

Dra. Maria Inês Martins é professora da PUC Minas (PUC-MG), Av. Dom José Gaspar 500 Prédio 1, Coração Eucarístico, BH, MG, 30535-610.ines@pucminas.br

Dra. Ana de Oliveira Rodrigues é professora do Centro Universitário de Belo Horizonte (UNI-BH) Av. Mário Werneck, 1685 , Estoril, BH, MG, 30455-610. ana@logicstyle.com.
Minas) are proposed as an alternative to the high cost procedures suggested by the standard. The results are validated through comparisons with analytical solutions. Measurements of shielding efficiency obtained using the proposed procedure show the difficulty in shielding low frequency fields and the consequent rise in the shielding efficiency as the frequency observed is increased. It is concluded that the proposed procedure can be used as a low cost alternative to the procedures presented in the international standard.

keywords - shielding, electromagnetic fields, measurements.

\section{INTRODUÇÃO}

Ondas eletromagnéticas são produzidas pela variação no tempo de um campo elétrico e um campo magnético. Para a região de campo distante no espaço livre, estes campos são perpendiculares entre si e perpendiculares à direção de propagação. A frequiência é o termo que define a taxa com que os sinais de telecomunicações e as correntes elétricas se alteram. É uma unidade de medida ligada a um fenômeno periódico que se repete num dado intervalo de tempo, e é dada pela Eq.1 e as faixas de freqüência são definidas conforme a Tabela I.

$$
f=\frac{v}{\lambda}[H z]
$$

onde: $f=$ freqüência $[\mathrm{Hz}], c=$ velocidade de propagção [m/s], $\lambda=$ comprimento de onda $[\mathrm{m}]$.

A freqüência é um dos principais fatores que caracterizam uma onda eletromagnética. Ondas eletromagnéticas irradiadas por equipamentos podem causar interferência eletromagnética, resultando em comportamento indevido em outros equipamentos, inclusive danificando-os. A área do conhecimento que estuda este tipo de interação denomina-se compatibilidade eletromagnética (CEM).

As soluções adotadas para evitar estes efeitos indesejáveis são diferentes se a fonte de interferência for de baixa freqüência e ou de alta frequiência. 
Tabela I

ESPECIFICAÇÃO DAS FAIXAS DE FREQÜÊNCIA [STIMAC, 2007]

\begin{tabular}{l|l|l}
\hline Sigla & Descrição & Faixa de Freqüência \\
\hline ULF & Freqüência Ultra Baixa & Menor que 3Hz \\
ELF & Frequiência Extra Baixa & $3-3000 \mathrm{~Hz}$ \\
VLF & Freqüência Muito Baixa & $3-30 \mathrm{kHz}$ \\
LF & Baixa Freqüência & $30-300 \mathrm{kHz}$ \\
MF & Média Freqüência & $0,3-3 \mathrm{MHz}$ \\
HF & Alta Freqüência & $3-30 \mathrm{MHz}$ \\
VHF & Freqüência Muito Alta & $30-300 \mathrm{MHz}$ \\
UHF & Freqüência Ultra Alta & $0,3-3 \mathrm{GHz}$ \\
SHF & Freqüência Super Alta & $3-30 \mathrm{GHz}$ \\
EHF & Freqüência Extremamente Alta & $30-300 \mathrm{GHz}$ \\
\hline
\end{tabular}

Geralmente a interferência eletromagnética pode ser atenuada pelo emprego de blindagens eletromagnéticas, cuja finalidade é proteger o interior de equipamentos dos efeitos dos campos eletromagnéticos exteriores ou proteger o meio ambiente dos efeitos dos campos eletromagnéticos gerados no interior dos equipamentos.

O material utilizado em uma blindagem eletromagnética deve apresentar bom índice de absorção, característica especialmente importante para baixa freqüência, bem como apresentar um bom índice de reflexão, característica especialmente importante para alta freqüência. Os materiais ferromagnéticos são os mais utilizados em ambos os casos.

Para testar a eficiência destas blindagens, devese adotar um procedimento para medição do campo eletromagnético com e sem a blindagem, validado para diversas frequiências através de medições laboratoriais. Estes procedimentos podem ser utilizados para avaliar se os níveis de irradiação observados estão em conformidade com as normas estabelecidas por lei e com os níveis aceitos por equipamentos.

Para o desenvolvimento destes procedimentos existe o padrão internacional IEEE Std-299-1997 [IEEE, 1997] e sua revisão, o IEEE Std-2992006 [IEEE, 2007]. Entretanto, este padrão exige a utilização de materiais de alto custo, nem sempre acessíveis à realidade brasileira.

\section{OBjetivos}

O objetivo deste trabalho é apresentar um procedimento para avaliação de blindagem eletromagnética através de medição de campos eletromagnéticos em laboratório.

Este procedimento foi baseado nas normas especificadas pelo padrão IEEE Std-299-1997, revisado em 2006, "IEEE Standard Method for Measuring the Effectiveness of Electromagnetic Shielding Enclosures" [IEEE, 1997] [IEEE, 2007], que serão adaptadas para a realidade dos laboratórios do Centro Universitário de Belo Horizonte (UNI-BH) e da Pontifícia Universidade Católica de Minas Gerais (PUC Minas).

Alternativas de baixo custo são oferecidas, com validação das medições obtidas.

\section{Metodologia}

\section{A. Calibração dos Equipamentos}

Os padrões IEEE Std 299-1997 [IEEE, 1997] e sua revisão, o padrão IEEE Std 299-2006 [IEEE, 2007] detalham a utilização de vários equipamentos para a medição da eficiência de blindagem eletromagnética.

A primeira etapa consiste em testes dos equipamentos para verificar se os medidores de campo elétrico e magnético para baixa freqüência estão funcionando corretamente. Pela lei de Ampère, o campo magnético gerado por um fio infinito, ou seja, quando comprimento do fio é muito maior do que a distância do fio ao ponto onde se quer saber o valor do campo magnético, é dado pela Eq.(2) [Hayt, 2003]:

$$
H_{\phi}=\frac{I}{2 \pi \rho}[A / m]
$$

onde: $I=$ corrente $[\mathrm{A}], \rho=$ distância ao fio $[\mathrm{m}]$.

Esta formulação assume que o fio é infinito. Para se obter maior precisão nos resultados, deve-se fazer o cálculo do campo magnético considerando o comprimento real do fio. A condição de fio finito é mostrada na Fig.1.

$\mathrm{O}$ campo magnético gerado pelo fio no ponto $\mathrm{P}$ é então dado pela Eq.(3) [Hayt, 2003], onde $\alpha_{1}$ e $\alpha_{2}$ são os ângulos apresentados na Fig.1 e estão em radianos:

$$
H_{\phi}=\frac{I}{4 \pi \rho}\left[\sin \left(\alpha_{2}\right)-\sin \left(\alpha_{1}\right)\right][A / m]
$$

Estas equações devem ser comparadas com valores medidos em laboratório com os medidores disponíveis, de forma a garantir a confiabilidade dos resultados. 


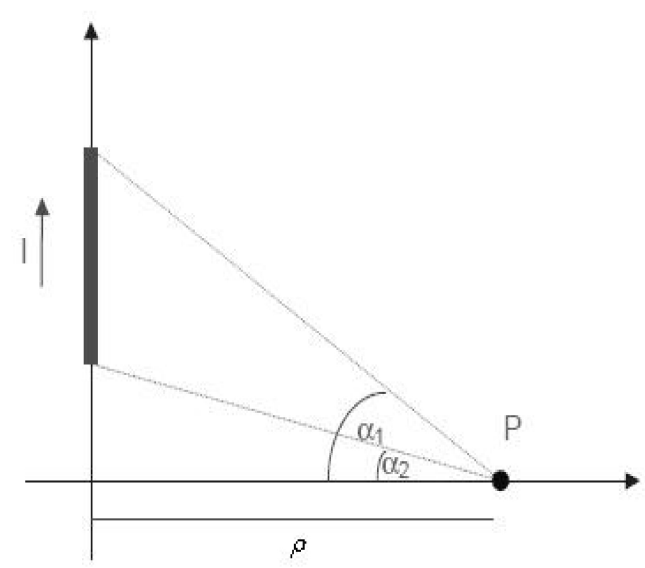

Figura 1. Configuração de um fio finito.

\section{B. Freqüências de Teste}

Dentro da faixa de $9 \mathrm{kHz}$ a $20 \mathrm{MHz}$, os padrões do IEEE recomendam a faixa de $14 \mathrm{MHz}$ a $16 \mathrm{MHz}$ para testes de campo magnético devido a menor possibilidade de falhas nesta faixa de frequiência. Além disto o padrão recomenda o teste em frequiências de interesse especial, como fontes conhecidas de interferência eletromagnética.

Utilizando esta recomendação e o alcance dos materiais disponíveis nos laboratórios, foi definido um conjunto de freqüências para teste:

a) $60 \mathrm{~Hz}$ (ELF) - freqüência da rede e possível causa de interferências eletromagnéticas causadas por equipamentos comerciais.

b) $12,5 \mathrm{KHz}$ (VLF) - valor intermediário da faixa VLF.

c) $150 \mathrm{KHz}(\mathrm{LF})$ - valor intermediário da faixa VF.

d) $15 \mathrm{MHz}(\mathrm{HF})$ - valor intermediário da faixa $\mathrm{HF}$.

e) $60 \mathrm{MHz}$ (VHF) - valor máximo obtido pelos equipamentos disponíveis no laboratório da PUC Minas.

f) $200 \mathrm{MHz}$ (VHF) - valor mínimo obtido pelos equipamentos disponíveis no laboratório do UNI-BH.

g) $450 \mathrm{MHz}(\mathrm{UHF})$ - valor intermediário da faixa UHF.

h) $900 \mathrm{MHz}$ (UHF) - valor de transmissão de telefonia celular analógica e possível causa de interferências eletromagnéticas.

i) $1,8 \mathrm{GHz}$ (UHF) - valor de transmissão de telefonia celular digital e possível causa de interferências eletromagnéticas.

Não foram escolhidos valores na faixa de média freqüência (MF - de $300 \mathrm{KHz}$ a $3 \mathrm{MHz}$ ) pois não haviam geradores e antenas nos laboratórios disponíveis para gerar sinais nesta faixa de frequiência.

\section{Medição de Blindagem em Baixa Freqüência}

Os padrões IEEE Std 299-1997 e IEEE Std 2992006 descrevem a metodologia para o cálculo da eficiência de uma blindagem para uma barreira simples (por exemplo, uma tela) [IEEE, 1997] e [IEEE, 2007].

Para esta faixa de frequiência, a fonte geradora de campo magnético (fora da blindagem) deve ser um loop de $30 \mathrm{~cm}$ de diâmetro conectado a um gerador de sinal para prover a corrente necessária no loop.

Inicialmente mede-se o campo magnético sem a colocação da blindagem, utilizando-se um loop receptor, também de $30 \mathrm{~cm}$ de diâmetro, conectado a um analisador de espectro. Este campo medido é chamado de campo de referência. A seguir, inserese a blindagem, e mede-se o campo dentro da área da blindagem captado pelo loop receptor.

Em ambas medições, os loops devem estar coplanares, com uma separação de $60 \mathrm{~cm}$ entre seus centros. Cada loop deve estar conectado ao seu respectivo dispositivo (gerador ou medidor) por cabo blindado, eletrostaticamente isolado. A Fig.2 mostra a correta localização dos dispositivos geradores e medidores de campo magnético.

O comportamento não-linear devido à alta permeabilidade ferromagnética da blindagem deve ser considerado antes de medir o desempenho da blindagem, já que um campo magnético muito intenso satura materiais magnéticos e causa erro na medida deste campo.

Este comportamento não linear pode ser determinado fazendo-se medições com o loop gerador e o receptor em função da intensidade da fonte. A potência de saída do gerador deve ser incrementada passo a passo, e as medidas do sinal recebido devem ser analisadas graficamente para a determinação do maior nível permitido para um desempenho linear. $\mathrm{Na}$ seção de resultados apresentamos este teste de linearidade.

Embora loops sejam dispositivos de baixo custo, o tamanho dos loops disponíveis não precisam ser necessariamente os exigidos pela norma. $\mathrm{Na}$ seção de resultados são apresentadas medições com diversos tamanhos de loop para demonstrar que a utilização de loops com tamanhos diferentes 


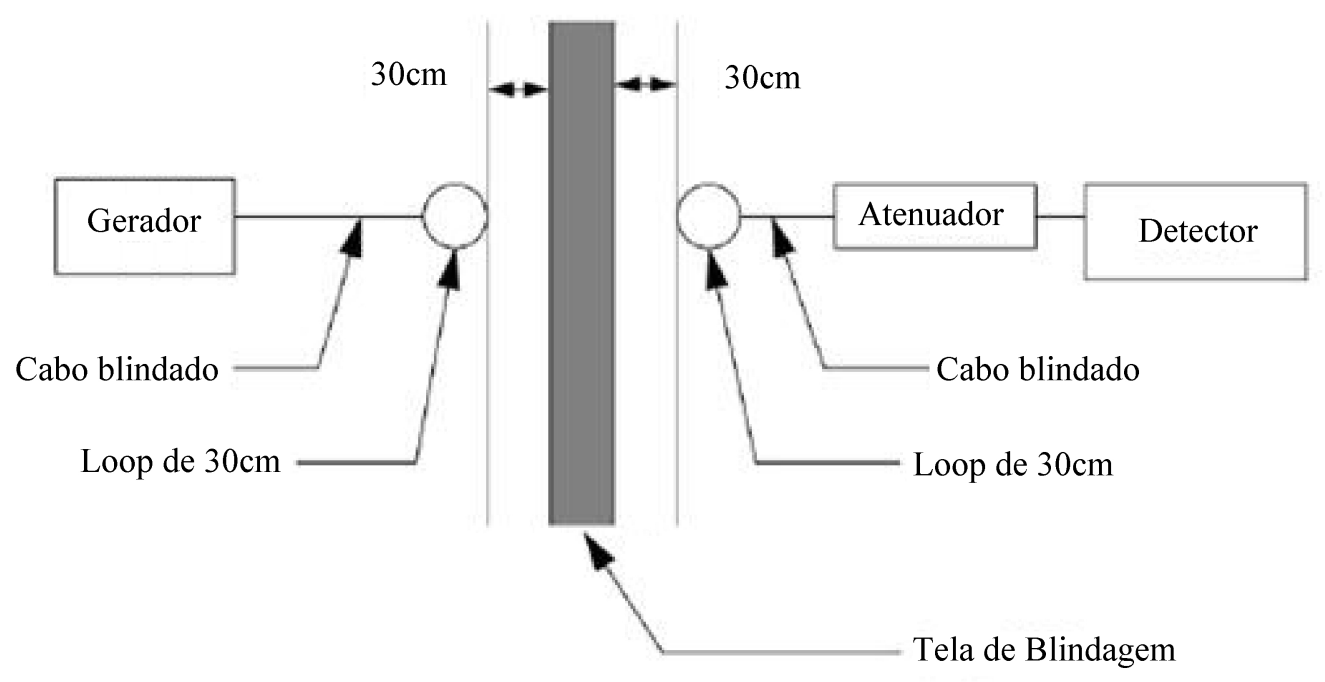

Figura 2. Localização correta dos instrumentos utilizados para medir a eficiência (SE) de uma blindagem. Adaptado de IEEE Std 299-2006.

da norma não interfere nas medições significativamente, evitando o custo de compra de novos equipamentos.

\section{Medição de Blindagem em Média Freqüência}

O padrão IEEE Std 1997 [IEEE,1997] e sua revisão em 2007 [IEEE, 2007] recomendam a utilização de antenas específicas para esta faixa de freqüência, assim como diversos cuidados que devem ser tomados na freqüência de ressonância (em MHz), dada pela Eq.(4):

$$
f r=150 \sqrt{\frac{1}{a^{2}}+\frac{1}{b^{2}}}[H z]
$$

onde: $a=$ altura da blindagem $[\mathrm{m}], b=$ largura da blindagem $[\mathrm{m}]$.

Para esse projeto, a freqüência de ressonância é então dada pela Eq.5:

$$
f r=150 \sqrt{\frac{1}{3,50^{2}}+\frac{1}{2,00^{2}}}=86,38 \mathrm{MHz}
$$

Nesta freqüência não devem ser feitas medições, pois os valores apresentam distorções devido à esta ressonância. Por isto, para a freqüência intermediária foi selecionada a blindagem apenas para a freqüência de $60 \mathrm{MHz}$. O custo das antenas específicas para esta frequiência é alto, então este trabalho apresenta medições com os loops utilizados nas medições para baixa freqüência.

\section{E. Medição de Blindagem em Alta Freqüência}

O padrão IEEE Std 1997 [IEEE,1997] e sua revisão em 2007 [IEEE, 2007] recomendam a utilização de antenas do tipo corneta (horn) para estas medições. Entretanto, o tamanho das antenas e das linhas de transmissão (guias de onda) para esta faixa de freqüência são dependentes da frequiência, o que implicaria na necessidade de se obter uma antena para cada frequiência medida.

A compra destes equipamentos apresenta alto custo, então este trabalho mostra a adaptação da norma com a utilização de duas antenas telescópicas, cujo comprimento seria ajustado em meio comprimento de onda $(\lambda / 2)$ para cada freqüência de interesse. Antenas telescópicas apresentam baixo custo e podem ser utilizadas para uma grande gama de frequiências, dependendo apenas dos valores de tamanho mínimo (totalmente fechada) e tamanho máximo (totalmente estendida).

Como mostrado anteriormente, definiu-se que as frequiências de medição para esta faixa de freqüência seriam $200 \mathrm{MHz}$ (VHF), $450 \mathrm{MHz}$ (UHF), $900 \mathrm{MHz}$ (UHF) e $1.8 \mathrm{GHz}$ (UHF). Estas são freqüências dentro da faixa que a antena telescópica disponível poderia fornecer, e abrangem as frequiências utilizadas pela telefonia celular $(900 \mathrm{MHz}$ e $1.8 \mathrm{GHz})$, fontes freqüentes de interferência eletromagnética.

A distância entre as antenas foi de $1 \mathrm{~m}$. A Tabela II mostra os parâmetros utilizados para cada frequiência de medição, onde $f$ é a freqüência, e $h_{r}$ é o comprimento da antena telescópica, igual a meio 
comprimento de onda $(\lambda / 2)$.

Tabela II

PARÂMETROS PARA MONTAGEM DAS MEDIÇÕES

\begin{tabular}{l|l|l}
\hline Medição & $f$ & $h_{r}$ \\
\hline 1 & $200 \mathrm{MHz}$ & $75 \mathrm{~cm}$ \\
2 & $450 \mathrm{MHz}$ & $33,33 \mathrm{~cm}$ \\
3 & $900 \mathrm{MHz}$ & $16,67 \mathrm{~cm}$ \\
4 & $1800 \mathrm{MHz}$ & $8,33 \mathrm{~cm}$ \\
\hline
\end{tabular}

\section{RESUltados}

\section{A. Testes dos Equipamentos}

1) Teste de Calibração: Para testar se o medidor de campo elétrico e magnético de baixa freqüência estava funcionando corretamente, mediu-se o campo magnético gerado por um fio de $1,57 \mathrm{~m}$ no qual circulava uma corrente de $3 \mathrm{~A}$. Os valores medidos foram comparados com valores calculados analiticamente, conforme detalhado na Metodologia. A Tabela III mostra esta comparação em função da distância.

Tabela III

VALORES DO CAMPO MAGNÉTICO CALCULADO PARA O FIO INFINITO, PARA O FIO FINITO E MEDIDO EM LABORATÓRIO

\begin{tabular}{c|c|c|c|c|c}
\hline \multirow{2}{*}{$\begin{array}{c}\rho \\
{[\mathrm{cm}]}\end{array}$} & \multicolumn{2}{|c|}{$\mathrm{H}$ calculado [A/m] } & H medido & \multicolumn{2}{c}{ Erro [A/m] } \\
\cline { 5 - 6 } & Infinito & Finito & {$[\mathrm{A} / \mathrm{m}]$} & Infinito & Finito \\
\hline 10 & 4,77 & 4,74 & 4,55 & 0,25 & 0,19 \\
15 & 3,18 & 3,13 & 2,90 & 0,28 & 0,23 \\
20 & 2,39 & 2,31 & 1,92 & 0,47 & 0,39 \\
25 & 1,91 & 1,82 & 1,38 & 0,53 & 0,44 \\
30 & 1,59 & 1,49 & 1,06 & 0,53 & 0,43 \\
35 & 1,36 & 1,25 & 0,84 & 0,52 & 0,41 \\
40 & 1,19 & 1,06 & 0,68 & 0,51 & 0,38 \\
45 & 1,06 & 0,92 & 0,57 & 0,49 & 0,35 \\
50 & 0,95 & 0,81 & 0,48 & 0,48 & 0,33 \\
55 & 0,87 & 0,71 & 0,42 & 0,45 & 0,29 \\
60 & 0,79 & 0,63 & 0,36 & 0,44 & 0,27 \\
\hline
\end{tabular}

Na Tabela III, percebe-se que o erro entre os valores medidos e calculados é menor quando considerase o fio como sendo finito. A Fig. 3 mostra os valores dos campos apresentados na Tabela III.

A Fig. 3 mostra que a curva característica foi a mesma para os valores calculados e os valores medidos. Observa-se que o erro na medição considerando o comprimento do fio é menor do que o erro quando o fio é considerado infinito. Observa-se que o erro cometido na medição é em torno $0,4[\mathrm{~A} / \mathrm{m}]$, e podemos atribuir sua existência a fatores tais como campo de fundo e calibração do medidor.
Assim, este erro passa a ser esperado nas medições. Como a eficiência será medida através da relação entre o campo medido com e sem blindagem, este erro estará presente em ambas as medições, não afetando o resultado final.

2) Teste de medição com diferentes loops: Os padrões do IEEE sugerem que o campo magnético seja gerado por um loop de $30 \mathrm{~cm}$ de diâmetro e não especifica quantas espiras ele deve ter, sendo que o loop medidor deve ser idêntico ao loop gerador.

No laboratório da PUC Minas estão disponíveis dois loops de $40 \mathrm{~cm}$ de diâmetro e um de $20 \mathrm{~cm}$ de diâmetro, além de um medidor de campo (HI-3604) que tem $20 \mathrm{~cm}$ de diâmetro. Devido à facilidade de transporte e utilização do HI-3604, há preferência em utilizá-lo para se medir a eficiência de uma blindagem eletromagnética para baixa frequiência. Para isto serão necessários testes com os loops, utilizando como geradores os loops de $40 \mathrm{~cm}$ e $20 \mathrm{~cm}$ de diâmetro, e utilizando como medidores o HI3604 e o loop de $40 \mathrm{~cm}$ de diâmetro. Estes testes verificarão se os resultados seriam compatíveis com os dos loops sugeridos pelos padrões.

O objetivo deste teste é mostrar que as dimensões físicas do HI-3604 não irão interferir no resultado final caso a medição seja feita com um loop de 20 cm de diâmetro, ou seja, tanto o medidor HI-3604 quanto um loop de $30 \mathrm{~cm}$ de diâmetro (recomendado pelos padrões do IEEE) podem ser utilizados. A distância entre os centros do loop gerador e do HI 3604 foi de $42 \mathrm{~cm}$. Esta distância foi escolhida pois coincide com o centro do loop receptor de $40 \mathrm{~cm}$ de diâmetro.

Utiliza-se a Eq.(6) [Hayt, 1993] para calcular o campo magnético deste loop e verificar se este campo é compatível com o campo medido pelo HI3604.

$$
H=\frac{N I}{2}\left(\frac{a^{2}}{\left(a^{2}+z^{2}\right)^{3 / 2}}\right)[A / m]
$$

$\mathrm{O}$ valor de campo magnético medido pelo HI3604 foi de $8 \mathrm{~A} / \mathrm{m}$. Com o auxílio de um amperímetro, determinou-se que o valor da corrente induzida no loop receptor foi de $84 \mu \mathrm{A}$. Assim, o campo magnético no centro deste loop é a diferença entre o campo magnético gerado pelos loops gerador e receptor.

Para o loop gerador o campo é dado pela Eq.(7), para o loop receptor o campo é dado pela Eq.(8), e 


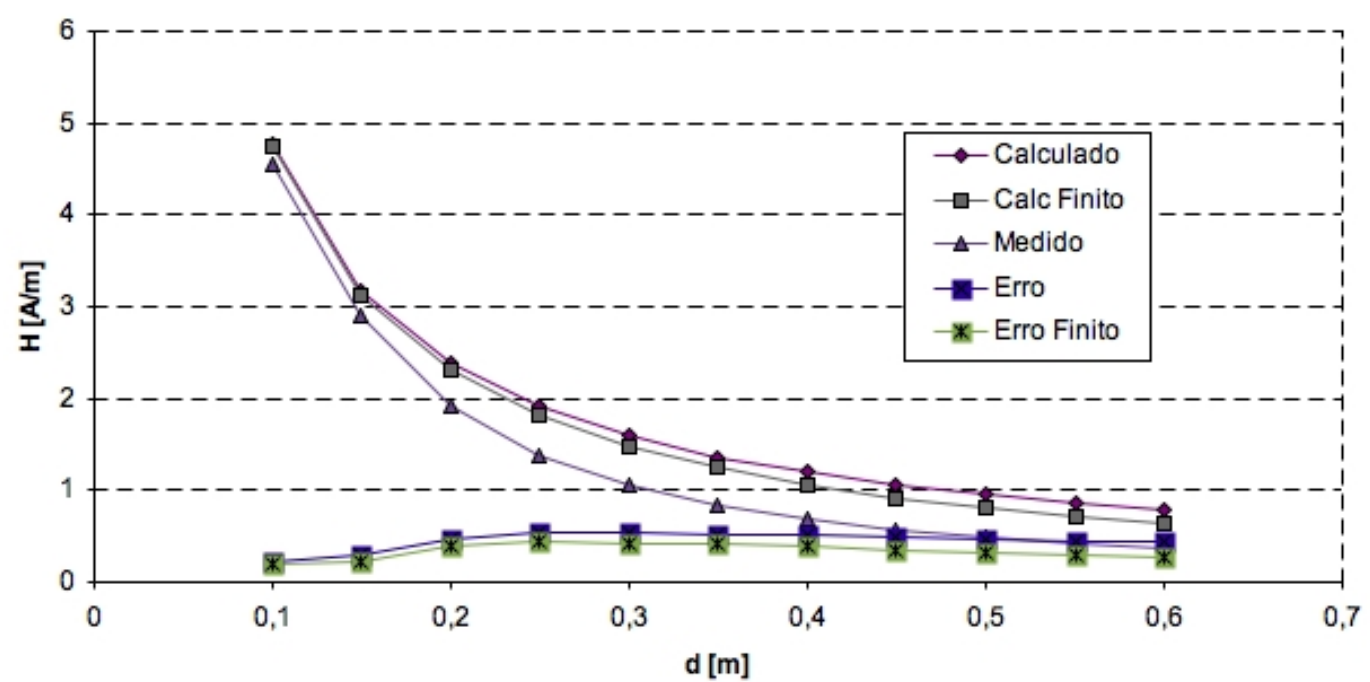

Figura 3. Valores de campo magnético

o campo magnético total é dado pela Eq.(9):

$$
\begin{aligned}
H & =\frac{10.4}{2}\left(\frac{0,2^{2}}{\left(0,2^{2}+0,42^{2}\right)^{3 / 2}}\right)=7,95 \\
H & =\frac{10.84 \mu}{2}\left(\frac{0,1^{2}}{\left(0,1^{2}+0^{2}\right)^{3 / 2}}\right)=0,0021 \\
H & =7,95-0,0021=7,95[\mathrm{~A} / \mathrm{m}]
\end{aligned}
$$

Percebe-se que se o campo magnético gerado pelo loop de $40 \mathrm{~cm}$ de diâmetro for medido usando um loop de $40 \mathrm{~cm}$ de diâmetro ou o medidor de campo HI-3604, o resultado será o mesmo. A próxima etapa será substituir o loop gerador de $40 \mathrm{~cm}$ de diâmetro por um de $20 \mathrm{~cm}$ de diâmetro e verificar se o resultado medido pelo HI-3604 é compatível com o resultado esperado.

A montagem feita neste teste é idêntica à anterior, com o loop gerador de $20 \mathrm{~cm}$ de diâmetro e a distância entre os centros dos loops de $22 \mathrm{~cm}$. O campo magnético gerado pelo loop gerador é dado pela Eq.(10) :

$$
H=\frac{N I}{2}\left(\frac{0,1^{2}}{\left(0,22^{2}+0^{2}\right)^{3 / 2}}\right)=14,17[A / m]
$$

O campo magnético medido pelo HI-3604 foi de $14,40 \mathrm{~A} / \mathrm{m}$. Percebe-se que os valores medidos e calculados estão muito próximos (erro de 1,6\%). Com isso, conclui-se que o HI-3604 é adequado para medir o campo gerado por estes loops para avaliação da blindagem eletromagnética.
3) Teste de linearidade: É necessário determinar qual a faixa em que o medidor se comporta linearmente (DR). Para isso foi feita outra montagem em laboratório, onde o campo foi sendo aumentado linearmente (através do aumento da tensão e, consequentemente, da corrente).

A Fig.4 mostra o valor calculado ("H calculado"), o valor real ("H real", que é o valor medido menos o valor de campo ambiente) e o erro observado. Observa-se que o erro é praticamente constante, em torno de $0,4[\mathrm{~A} / \mathrm{m}]$, como observado anteriormente no teste de funcionamento. Este teste do DR mostra que o comportamento do medidor é linear dentro da faixa de teste utilizada, podendo ser utilizado para as medições pretendidas.

\section{B. Resultados em Baixa e Média Freqüência}

Para medir a eficiência de uma blindagem eletromagnética em baixa e média frequiência, como detalhado na metodologia, as freqüências escolhidas foram $60 \mathrm{~Hz}$ (VLF), $12,5 \mathrm{kHz}$ (VLF), $150 \mathrm{KHz}(\mathrm{LF})$, $15 \mathrm{MHz}(\mathrm{HF})$ e $60 \mathrm{MHz}$ (VHF).

As medições foram realizadas no laboratório de eletromagnetismo da PUC-MG, seguindo as orientações dos padrões IEEE Std-299 e IEEE Std299. Embora estes padrões orientem para que os loops sejam de $30 \mathrm{~cm}$ de diâmetro, foi mostrado anteriormente que o resultado será o mesmo se forem utilizados os loops de $20 \mathrm{~cm}$ disponíveis no laboratório. A Fig.5 mostra a montagem realizada no laboratório, sem e com a blindagem (tela). 


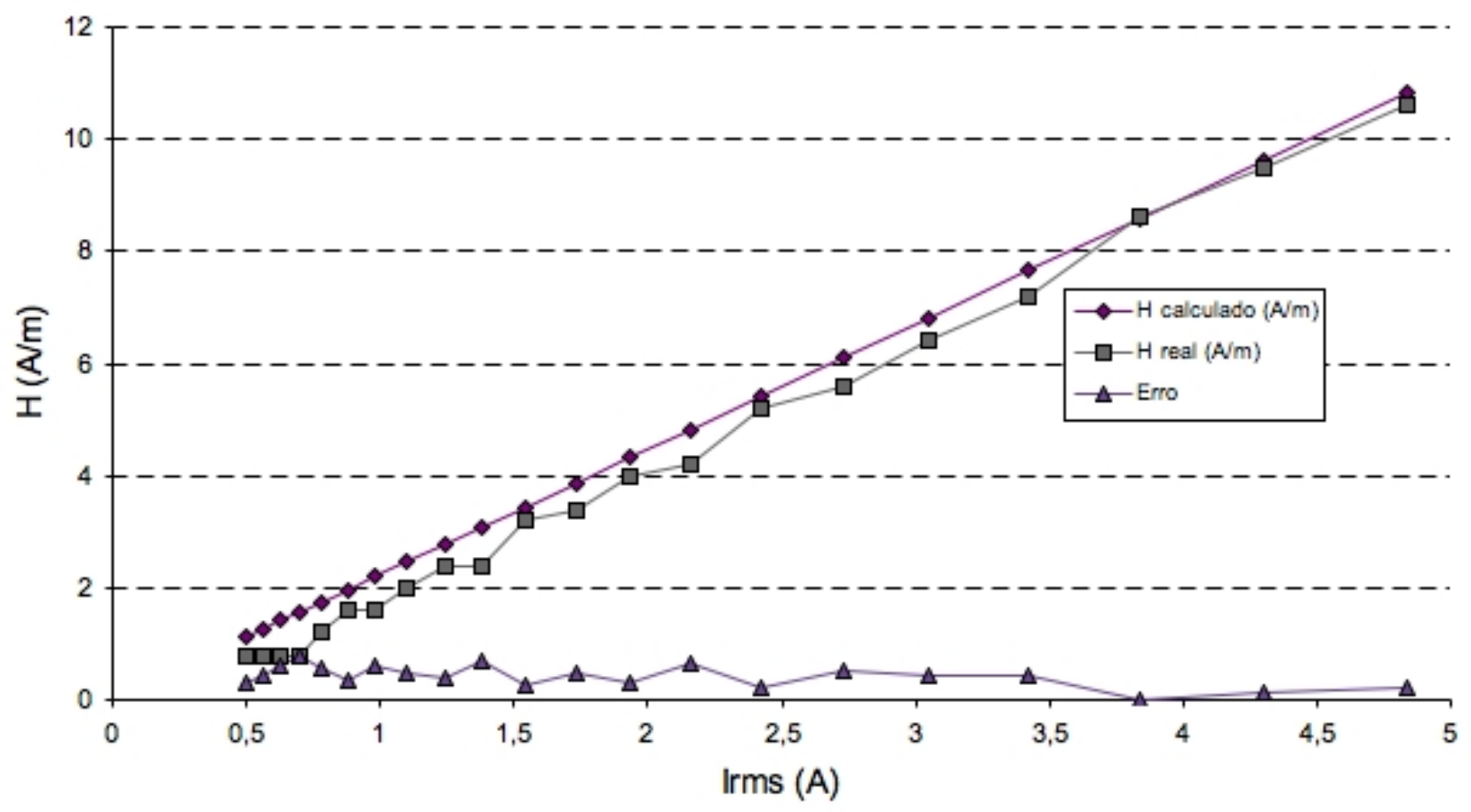

Figura 4. Teste de linearidade
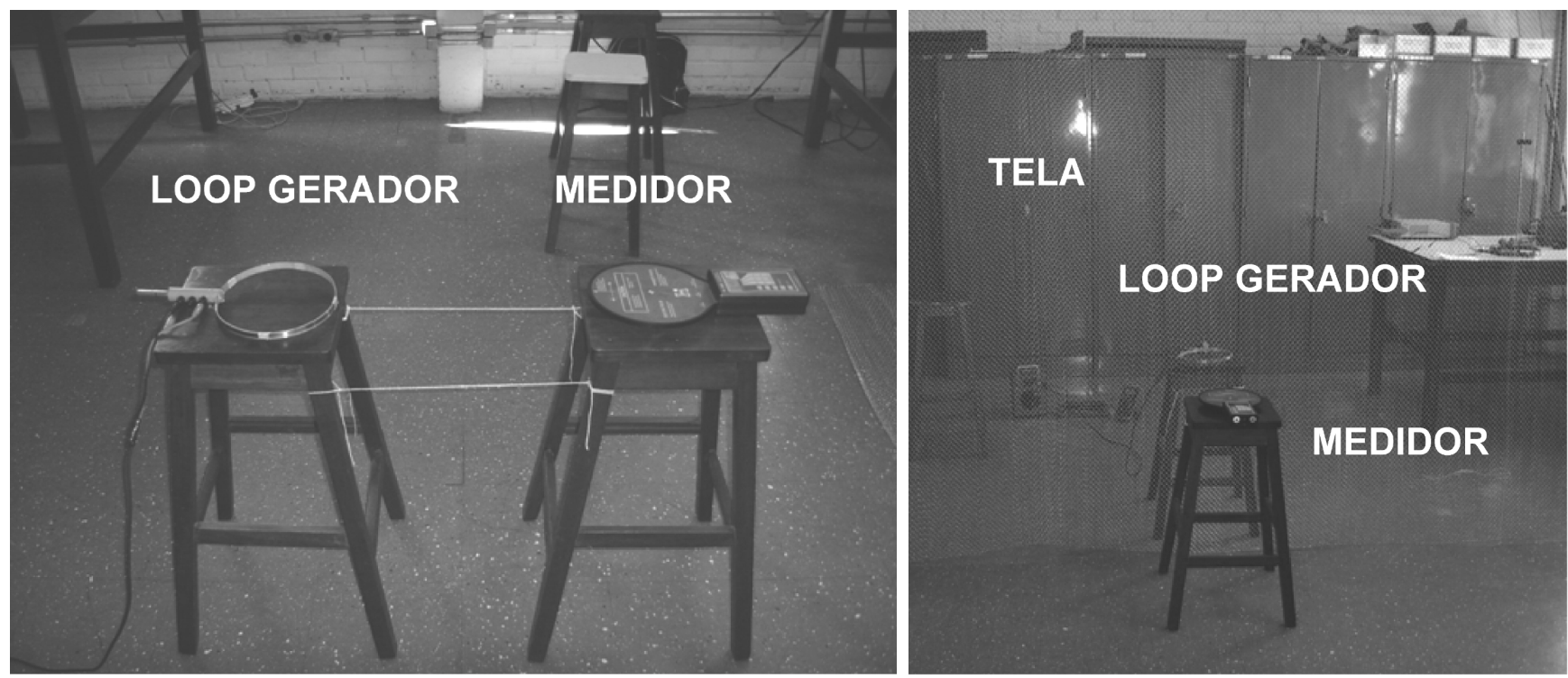

Figura 5. Medição do campo residual e da blindagem em $60 \mathrm{~Hz}$

A medição de campo foi feita inicialmente com o gerador de sinal desligado, para medir valor de campo residual no ambiente. $\mathrm{O}$ valor de campo medido pelo HI-3604 foi $0,048 \mathrm{~A} / \mathrm{m}$ que é aproximadamente o valor de erro do medidor detectado nos testes anteriores. Este erro não influenciará no resultado final, pois o foco é a relação entre os campos com e sem a blindagem.

Foi então aplicado ao loop gerador (10 espiras) uma corrente de $4 \mathrm{~A}$ e o valor de campo de referência lido foi de $0,525 \mathrm{~A} / \mathrm{m}$. A blindagem a ser medida foi criada através do posicionamento de uma tela metálica de 2 metros de altura e 3,50 metros de largura entre as antenas transmissora e receptora, como mostra a Fig.5.

O campo magnético lido após a blindagem foi de 0,511A/m. Assim, a eficiência da blindagem é dada 


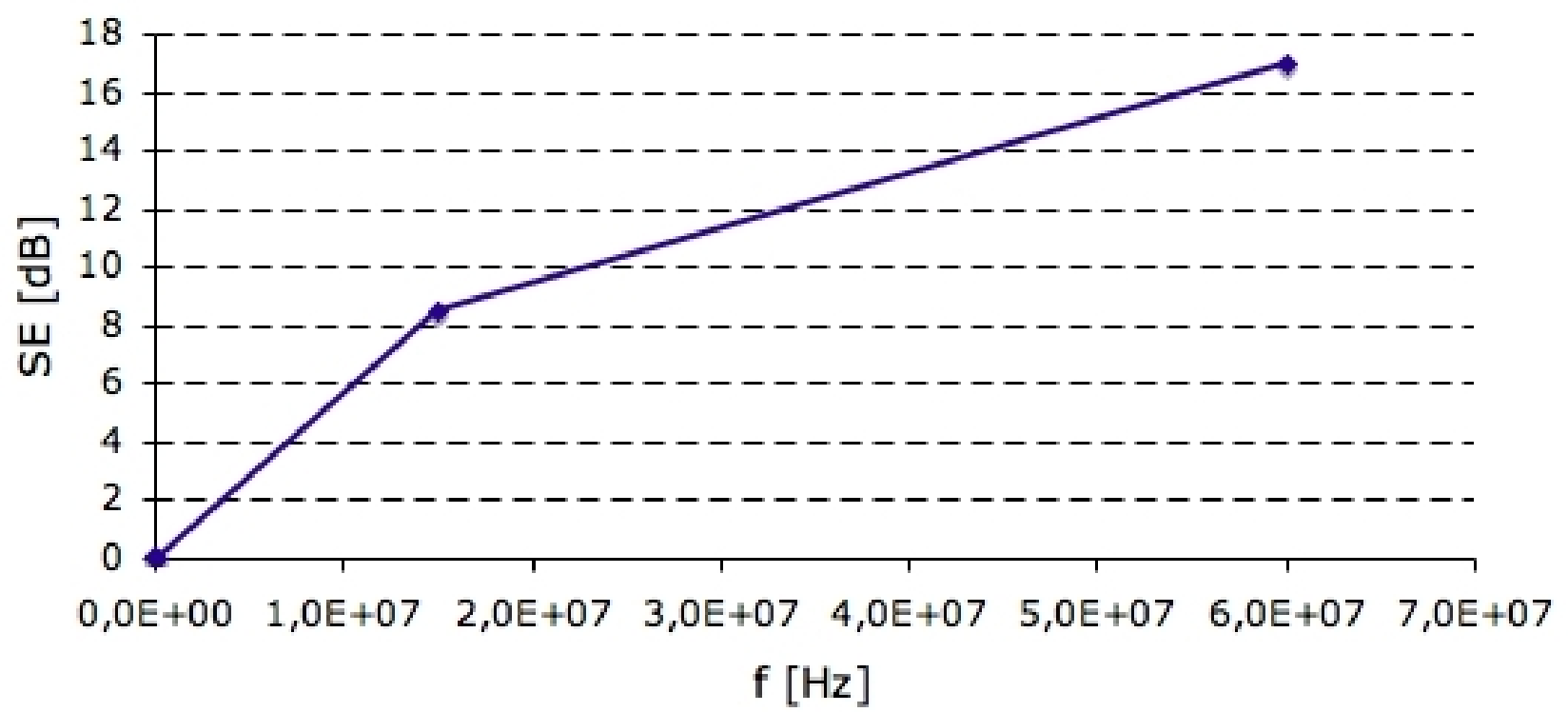

Figura 6. Blindagem em baixa freqüência e freqüência intermediária

pela Eq.(11) [IEEE, 1997] [IEEE, 2007].

$$
\begin{aligned}
S E & =20 \log \left(\frac{H_{1}}{H_{2}}\right)=20 \log \left(\frac{0,525}{0,511}\right) \\
& =0,23 d B
\end{aligned}
$$

Para as frequiências de $12,5 \mathrm{kHz}, 125 \mathrm{kHz}, 15 \mathrm{MHz}$ e $60 \mathrm{MHz}$ os procedimentos foram idênticos ao anterior, mas com a necessidade de trocar o loop receptor por um de $20 \mathrm{~cm}$ de diâmetro, 10 espiras e conectá-lo a um analisador de espectro. A mudança do loop receptor foi necessária pois a faixa de resposta em frequiência do $\mathrm{HI}-3604$ vai de $50 \mathrm{~Hz}$ a $1 \mathrm{kHz}$ [Holaday].

Os resultados das medições estão resumidos na Tabela IV, onde são apresentadas a magnitude do sinal em $\mathrm{dBm}$ sem e com blindagem e a respectiva eficiência da blindagem (SE).

Tabela IV

BLINDAGEM EM $60 \mathrm{~Hz}, 12,5 \mathrm{KHz}, 150 \mathrm{KHz}, 15 \mathrm{MHz}$ E $60 \mathrm{MHz}$

\begin{tabular}{l|l|l|l}
\hline Freqüência & Sem Tela & Com Tela & SE \\
\hline $60 \mathrm{~Hz}$ & $-27,12 \mathrm{dBm}$ & $-27,09 \mathrm{dBm}$ & $-0,03 \mathrm{~dB}$ \\
$12,5 \mathrm{kHz}$ & $-54 \mathrm{dBm}$ & $-53,9 \mathrm{dBm}$ & $-0,11 \mathrm{~dB}$ \\
$150 \mathrm{kHz}$ & $-61,5 \mathrm{dBm}$ & $-61,5 \mathrm{dBm}$ & $0 \mathrm{~dB}$ \\
$15 \mathrm{MHz}$ & $-36,5 \mathrm{dBm}$ & $-45 \mathrm{dBm}$ & $-8,5 \mathrm{~dB}$ \\
$60 \mathrm{MHz}$ & $-28 \mathrm{dBm}$ & $-45 \mathrm{dBm}$ & $-17 \mathrm{~dB}$ \\
\hline
\end{tabular}

A Tabela IV mostra que a tela começa a atenuar de forma significativa apenas a partir de $15 \mathrm{MHz}$. Este efeito de dificuldade de blindagem de campos de baixa freqüência é esperado, inclusive pelo fato de que abaixo desta freqüência a tela encontra-se em região de campo próximo [Balanis, 1992]. A Fig.6 apresenta um gráfico com os valores medidos para baixa freqüência e freqüência intermediária.

\section{Resultados em Alta Freqüência}

Duas antenas telescópicas foram alinhadas no laboratório de Eletromagnetismo do Centro Universitário de Belo Horizonte (UNI-BH). Os equipamentos utilizados foram: duas bases giratórias da marca Bit9; um analisador de espectro IFR modelo 2397, um gerador Agilent modelo 8648 e duas antenas telescópicas. A blindagem a ser medida foi gerada pelo posicionamento de uma tela metálica de $1,0 \mathrm{~m}$ de altura por 2,3m de largura, tipo peneira, entre a antena transmissora e a antena receptora.

As medições foram realizadas em quatro diferentes frequiências, $200 \mathrm{MHz}, 450 \mathrm{Mhz}, 900 \mathrm{MHz}$ e $1.8 \mathrm{GHz}$. Em todas as medições as antenas telescópicas estavam alinhadas no mesmo nível e possuíam o mesmo comprimento. A antena transmissora foi conectada ao gerador que irradiava a uma potência de $10 \mathrm{dBm}$ e a antena receptora foi conectada ao analisador de espectro para medição da potência. A Fig.7 apresenta a montagem com a tela.

A Fig.8 apresenta os resultados das medições gravadas diretamente pelo analisador de espectro sem a tela (esquerda) e com a tela (direita), mostrando 


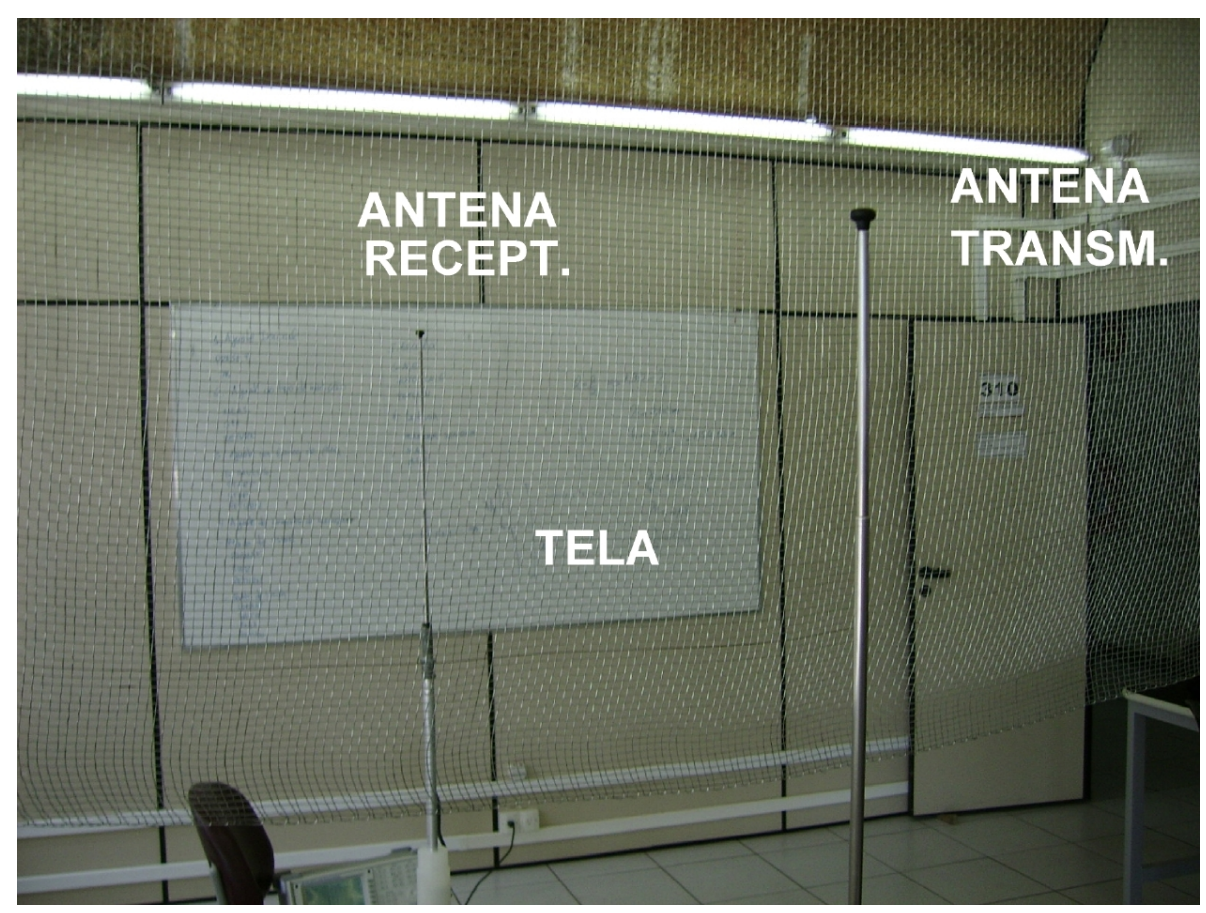

Figura 7. Medição da blindagem em alta frequiência
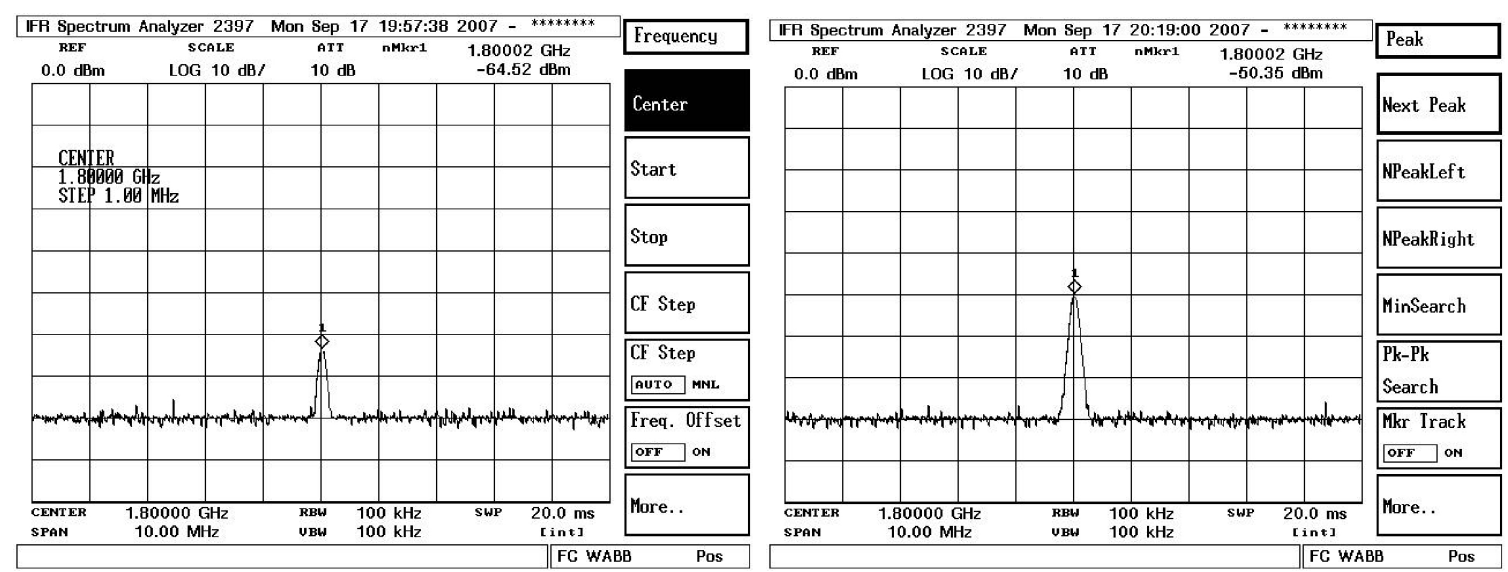

Figura 8. Efeito da blindagem na freqüência de $1.800 \mathrm{MHz}$

o efeito da blindagem em $1800 \mathrm{MHz}$. Os resultados para $200 \mathrm{MHz}, 450 \mathrm{MHz}$ e $900 \mathrm{MHz}$ são similares.

A Tabela V e a Fig.9 detalham os resultados obtidos, mostrando a variação da eficiência da blindagem (SE) com a frequiência. Novamente, observase o aumento da eficiência da blindagem com o aumento da frequiência.

\section{Discuss Ão dos Resultados}

Os resultados obtidos mostram que os testes feitos nos equipamentos as medições de eficiência de blindagem eletromagnética com o procedimento desenvolvido apresentam resultados coerentes.
Tabela V

BLINDAGEM EM ALTA FREQÜÊNCIA

\begin{tabular}{l|l|l|l}
\hline Frequiência & Sem Tela & Com Tela & SE \\
\hline $200 \mathrm{MHz}$ & $-55,91 \mathrm{dBm}$ & $-62,50 \mathrm{dBm}$ & $-6,59 \mathrm{~dB}$ \\
$450 \mathrm{MHz}$ & $-35,33 \mathrm{dBm}$ & $-45,12 \mathrm{dBm}$ & $-9,79 \mathrm{~dB}$ \\
$900 \mathrm{MHz}$ & $-42,15 \mathrm{dBm}$ & $-55,00 \mathrm{dBm}$ & $-12,85 \mathrm{~dB}$ \\
$1800 \mathrm{MHz}$ & $-50,35 \mathrm{dBm}$ & $-64,52 \mathrm{dBm}$ & $-14,17 \mathrm{~dB}$ \\
\hline
\end{tabular}

Os testes mostram que o campo ambiente pode ser medido e desconsiderado das medições, evitando o uso de câmaras anecóicas de alto custo. As comparações com soluções analíticas validam as medições com equipamentos simples como loops, evitando o uso de equipamentos de alto custo para 


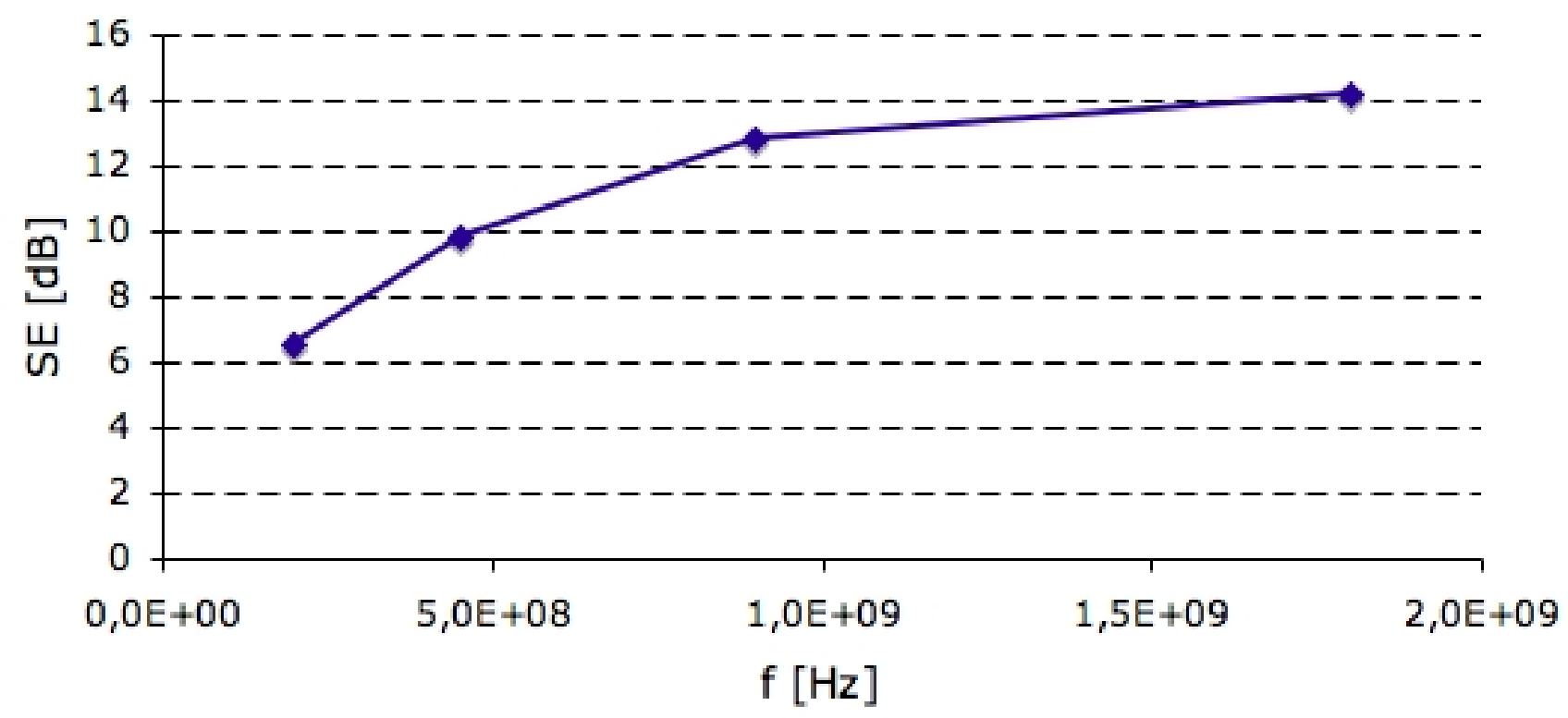

Figura 9. Blindagem em alta freqüência

a geração e medição do campo eletromagnético.

Nota-se a dificuldade de blindagem de campos de baixa freqüência, e conseqüente aumento da eficiência da blindagem com o aumento da freqüência.

\section{CONCLUSÃo}

Foi apresentado um procedimento para avaliação de blindagem eletromagnética através de medição de campos eletromagnéticos em laboratório.

Este procedimento foi baseado nas normas especificadas pelos padrões IEEE Std-299-1997 e sua revisão em 2006 [IEEE, 1997] [IEEE, 2007], adaptados para a realidade dos laboratórios do Centro Universitário de Belo Horizonte (UNI-BH) e da Pontifícia Universidade Católica de Minas Gerais (PUC Minas).

Alternativas de baixo custo aos materiais e métodos sugeridos pela norma são oferecidas, como a utilização da mesma antena para medições em baixa freqüência e freqüência intermediária e a substituição de antenas do tipo corneta por antenas telescópicas com custo muito menor.

Foram apresentadas as validações das medições através de comparação com soluções analíticas. O comportamento da blindagem encontra-se coerente e as curvas estão dentro dos padrões esperados para campos de baixa e alta freqüência, indicando que o procedimento desenvolvido mostra-se como uma alternativa de baixo custo aos métodos apresentados nos padrões internacionais.

\section{AgRADECIMENTOS}

Os autores gostariam de agradecer ao Centro Universitário de Belo Horizonte, (UNI-BH) e à Pontifícia Universidade Católica de Minas Gerais (PUC Minas) pela disponibilização de equipamentos e dos laboratórios de Eletromagnetismo onde as medições foram feitas, e pelo apoio ao desenvolvimento desta pesquisa.

\section{REFERÊNCIAS BIBLIOGRÁFICAS}

[Balanis, 1992] C. A. Balanis. Antenna theory: analysis and design. J. Wiley \& Sons, New York, NY. 1992.

[Hayt, 2003] John A Buck Willian H. Hayt, jr. ELETROMAGNETISMO sexta edição. LTC Editora, Rio de Janeiro, RJ. 2003.

[Holaday] Holaday Industries Inc. Hi-3604 User's manual.

[IEEE, 1997] IEEE Std 299-1997: IEEE Standard Method for Measuring the Effectiveness of Electromagnetic Shielding Enclosure. IEEE Power Engineering Society Sponsored by the IEEE Electromagnetic Compatibility Committee. 1997.

[IEEE, 2007] IEEE Std 299-2006 (Revision of IEEE Std 299-1997) : IEEE Standard Method for Measuring the Effectiveness of Electromagnetic Shielding Enclosure. IEEE Power Engineering Society Sponsored by the IEEE Electromagnetic Compatibility Committee. 2007.

[Stimac, 2007] Tomislav Stimac. Definition of frequency bands (VLF, ELF... etc.). Disponível online em http://www.vlf.it/frequency/bands.html. 09/2007. 